porate Paramecium axonemal tubulin. After fixation, the exogenous tubulin could be located, using an axonemal tubulin-specific antibody, against the background of endogenous microtubules labelled with a more general probe. In this way, axonemal tubulin was seen at late anaphase to be incorporated as a thin equatorial ring. Double immunofluorescence showed that incorporation occurred at the ends of existing endogenous microtubules. Later, in telophase when spindle pole microtubules were less abundant, there was a more extensive incorporation of exogenous tubulin throughout the phragmoplast. Again, initial labelling of a discrete equatorial ring, followed by increased labelling throughout the breadth of phragmoplast microtubules, indicates some special activity of the interzonal region.

Complementary studies ${ }^{6}$ on the microinjection of fluorescent phalloidin into Haemanthus endosperm cells confirm the special nature of this region in cytoskeletal terms. Label injected at the beginning of mitosis is incorporated into actin filaments that surround the nucleus or run within the spindle, parallel to microtubule fibres. No label is incorporated into the phragmoplast, although this structure can be labelled by a second microinjection at late anaphase or telophase. This implies that the phragmoplast 'wreath' of F-actin filaments is a newly polymerized structure rather than being formed from redistribution of the filaments stretched between the poles at metaphase. An incidental aspect of such studies from Lambert's laboratory, which indicates that actin antagonists impede chromosome separation, is the challenging possibility that $\mathrm{F}$-actin might play some part in plant mitosis.

It will be important to establish whether there is continuity between spindle and phragmoplast microtubules or whether there is a new phase of nucleation at the mid-zone. The possibility of microtubule motors (as a cause of microtubule movement within the phragmoplast) is enticing, although it is too early to say whether or not tubulin incorporated at the interdigitating plus ends might move through microtubules by flux, or whether minus-end assembly is involved. In more general terms, the encouraging message of these recent papers is that incorporation of probes into the plant cytoskeleton is feasible, and that it is now possible to study turnover and movement in living plant cells. Given the centrality of microtubules to plant morphogenesis we can expect exciting developments as such studies are extended to interphase and pre-mitotic cells

Clive Lloyd is in the Department of Cell Biology, John Innes Institute, Colney Lane, Norwich NR4 TUH, UK.

1. Asada, T., Sonobe, S. \& Shibaoka, H. Nature 350, 238-241 (1991).

2. Euteneuer, U. \& McIntosh, J.R. J. Cell Biol. 87. 509 (1980) 2. Euteneuer. U.\& Mclntosh, J.R. J. Cell Biol. 87.509)

4. Zhang, D., Wadsworth, P. \& Hepler, P. K. Proc. natn. Acad. Sci. U.S.A. 87. 8820-8824 (1990)

5. Vantard, M., Levilliers, N., Hill, A.-M., Adoutte, A. \& Lambert,

A.-M. Proc. natn. Acad. Sci. U.S.A. 87, 8825-8829(1990)

6. Schmit, A. C. \& Lambert, A.-M. Pl. cel/ 2. 129-138(1990)

\title{
Seven genes for three diseases
}

\author{
Richard D. Wood
}

NEw twists to the connection between DNA repair and cancer emerged at a meeting last month*, with reports that the relationship between two inherited repair disorders having very different cancer risks is much closer than previously thought. Moreover, the relationship of these syndromes to a third unusual condition was strengthened.

People suffering from the skin tumourprone disease xeroderma pigmentosum (XP) are highly susceptible to sunlight, and their cells display deficiencies in the excision from DNA of damage caused by ultraviolet light. The XP complementation groups A to $G$ represent different genes in the excisionrepair pathway, and mutations in any one can give rise to some form of the disease. Now it seems that Cockayne's syndrome (CS), which was previously considered to be quite distinct, may arise from mutations in different components of the XP-repair system. And another disease, trichothiodystrophy, which causes brittle hair, shows tight genetic linkage with an XP gene. Studies of the particular muiations in the seven or more genes that can lead to these three conditions may provide a model for other pathogenic conditions arising from alterations in proteins that cooperate to form a functional complex. Unexpectedly, the new data have made it clear that some individuals harbour a severe DNA-repair deficiency without an increased risk of skin cancer.

In the more common forms of Cockayne's syndrome, patients show sensitivity to sunlight, but the freckling, dermatosis and cancerous lesions of xeroderma pigmentosum are absent. Instead, other distinctive physical and neurological defects are displayed. Cells from individuals suffering from Cockayne's syndrome seem to have a subtle defect in excision repair that is restricted to actively transcribed genes ${ }^{1}$. One connection between the two diseases was already known: the only well-charact srized individual from $X P$ group B hard symptoms of both diseases (see the discussion in News and Views last year ${ }^{2}$ ).

Recently, at least six more patients with both xeroderma pigmentosum and Cockayne's syndrome have been identified. Two have been assigned to XP group B, but, surprisingly, three fall into group $\mathrm{G}$ and one into group D (D. Bootsma, Erasmus University, Rotterdam; A. Lehmann, University of Sussex). Another patient with both diseases was previously allocated to the spurious group $\mathrm{H}$, but has now been assigned to group $\mathrm{D}$ (independent evidence from Bootsma; $\mathrm{R}$. Johnson, University of Cambridge; M. Stefanini, CNR, Pavia). But not all patients with CS show symptoms of XP, nor do all XP sufferers show signs of CS. It appears that allelic differences between mutations in this family of genes can lead to one disease, or the other, or some combination of both.
The hallmarks of the third disease, trichothiodystrophy ${ }^{3}$, are brittle, sulphur-deficient hair, mental and physical retardation, abnormal nail growth and scaly skin. About half of 21 patients that have been carefully studied are photosensitive, and they show defects in DNA repair that correspond to XP group D by complementation analysis (Stefanini). Others afflicted with trichothiodystrophy clearly do not have xeroderma pigmentosum, and so the intriguing relationship is most easily explained by there being a very close genetic linkage of two genes. No doubt a more precise biochemical definition of the altered sulphur metabolism in trichothiodystrophy would help in resolving this issue.

Neither the XP-D/trichothiodystrophy patients nor the two new XP-B patients (in their late thirties) have skin tumours. This sort of observation is directing attention to immunological surveillance systems in xeroderma pigmentosum, and their possible role in the aetiology of solar tumorogenesis in the disease ${ }^{4,5}$. Provocatively, six out of the seven patients with xeroderma pigmentosum examined so far in one study showed about one-third of normal natural killer-cell activity; the exception was an individual who had experienced multiple, self-healing melanomas over the course of several decades, suggesting the existence of an especially efficient anti-tumour defence system in this patient. People with Cockayne's syndrome and trichothiodystrophy but without XP symptoms or tumours had normal immune function (P. Norris, St Thomas's Hospital, London). Other factors, including pigmentation, certainly can influence the occurrence of skin cancers. For example, such carcinomas are very rare in Japan, even in individuals suffering from xeroderma pigmentosum who have severe repair defects $(\mathrm{H}$. Takebe, Kyoto University).

The existence of people with mild xeroderma pigmentosum and a marked DNA-repair deficiency but no tumours might suggest that the proportion of asymptomatic carriers of DNA-repair alterations is larger than previously suspected. If this is indeed the case, our views of the effects of environmental mutagens and carcinogens on the general population could require substantial reassessment.

Richard D. Wood is at the Imperial Cancer Research Fund Clare Hall Laboratories, South Mimms, Hertfordshire EN6 3LD, UK.

1. Venema, J., Mullenders, L. H. F., Natarajan, A. T., Van Zeeland. A. A. \& Mayne, L. V. Proc. natn. Acad. Sci. U.S.A. 87 4707-4711 (1990).

. Wood, R. D. \& Lindahl, T. Nature 348, 13-14 (1990)

3. Lehmann, A. R. et al. Cancer Res. 48, 6090-6096 (1988) 4. Bridges, B. A. Carcinogenesis 2, 471-472 (1981) 5. Norris, P. G. et al. J. invest. Dermatol. 94, 94-100 (1990).

* A New Look at Excision Repair Disorders, Eastbourne, UK. 27 February-1 March 1991. 